\title{
The Effects of Gamma Irradiation on RTV Polysiloxane Foams.
}

\author{
Andrea Labouriau*, Carl Cady, John Gill, Dean Taylor, Adam Zocco, Jamie Stull, \\ Kevin Henderson and Debra Wrobleski \\ Los Alamos National Laboratory, Los Alamos, New Mexico, 87545
}

\begin{abstract}
:
The present work involves the investigation of ionizing radiation effects on silica filled poly (dimethyl siloxane) foam vulcanized at room temperature. In order to better predict aging effects in these materials, it is important to understand the influence of irradiation on structural-rheological property relationships. Polysiloxane foams were subjected to moderate doses of gamma irradiation in an inert atmosphere and characterized by thermal (DSC and TGA), chemical (FT-IR, NMR, Mössbauer, mass spectroscopy, EPR, solvent swelling), microscopy (SEM and AFM), and mechanical (uniaxial compressive load) techniques. Radiation exposure induced cross-linking reactions that predominated over chain scission reactions for the dose range investigated. No long-lived radiation-induced radicals were detected and the porous structure of the irradiated foam remained unchanged. Radiation exposure resulted in gas evolution, decrease in crystallization levels, slight changes in chemistry, and decrease in the molecular weight between cross-links, thereby hardening the foam.
\end{abstract}

\section{LA-UR-14-28787}

Keywords: polysiloxanes, gamma irradiation, RTV, foams

Corresponding author: A. Labouriau (andrea@lanl.gov), phone: 1-505-667-7216. 


\section{Introduction.}

The structural changes that occur in poly (dimethyl siloxane) (PDMS) due to exposure to high energy irradiation have been the subject of several studies over the years. Charlesby, amongst others, reported formation of a cross-linked network accompanied by gas evolution. ${ }^{1-3}$ Enhancements on tensile properties and increasing hardness after radiolysis were observed by Warrick and others. ${ }^{4,5}$ However, limited work was performed on the effects of gamma irradiation on the properties of polysiloxane foams. A set of studies by Maxwell and co-workers investigated silica filled polysiloxane foams (RTV-5370) exposed to gamma doses that varied from $30 \mathrm{kGy}$ to $3.8 \mathrm{MGy}$ in an air atmosphere. ${ }^{6,7}$ With increasing radiation dose, the authors observed a decrease in chain segmental mobility as measured by NMR relaxation times, hardening of the foam as evidenced by dynamic mechanical analysis (DMA) measurements and changes in crystallization levels and melting temperature supported by DSC results. In addition, EPR results showed evidence of trapped paramagnetic species within the silica filler matrix, whereas solvent swelling experiments and NMR studies revealed reduction of the polymer filler interaction at low doses. Nonetheless, this did not greatly affect the mechanical properties of the material. In another study on the radiation resistance of polysiloxane foams containing phenyl groups, Wei and co-workers detected changes in mechanical response, gas evolution and collapse of the microstructure at large gamma exposures. ${ }^{8}$ Also, gamma irradiation studies at doses up to $10 \mathrm{kGy}$ performed on a set of polysiloxane foams (RTV-5370) showed that the side of the foam exposed to gamma irradiation exhibited minor changes at the surface when aged with water, as monitored by attenuated total reflectance (ATR)-FTIR spectroscopy coupled with multivariate curve resolution analysis. ${ }^{9}$

In this work, we examined the effects of exposing silica-reinforced polysiloxane foams to moderate doses of gamma irradiation (less than $250 \mathrm{kGy}$ ). The foam of interest is known as SX358 and is related to the RTV-5370 foam investigated in earlier studies. Both foams are cured at room temperature and have similar chemistry, except that the cross-link density in the SX358 foam is half that of RTV-5370. Another important distinction between the present work and those performed for RTV-5370 is that SX358 foams were exposed to ionizing irradiation under 
nitrogen atmosphere, instead of air. It is well known that the atmosphere under which the polymer is irradiated has an important effect on the reactions that take place. Under inert atmosphere radicals tend to react to form cross-links, while under reactive atmosphere like air, oxygen reacts with free radicals and initiates oxidative degradation, which generally causes material weakening. In particular, the presence of oxygen may lead to formation of peroxides which can compete with cross-link reactions. ${ }^{10,11}$ In the present work, radiation-induced changes in chemistry, thermal properties, phase behavior, microstructure, and mechanical response were assessed by mass spectroscopy, solid and solution-state nuclear magnetic resonance (NMR), Mössbauer spectroscopy, electron paramagnetic resonance (EPR), solvent swelling, differential scanning calorimetry (DSC), thermogravimetric analysis (TGA), image techniques, and mechanical compression testing. The combination of all these diverse methods provided useful insights on the radiolysis in inert atmospheres of room temperature vulcanizing (RTV) polysiloxane foams. 


\section{Materials and Methods.}

2.1. Materials. SX358 silicone foam was synthesized from a polysiloxane elastomeric gumstock that contained 75 wt\% hydroxyl-terminated polydimethylsiloxane (PDMS, 3 wt\% $\begin{array}{lllllll}\text { polymethylhydrosiloxane } & \text { (PMHS), } 2 \text { wt\% } & \text { tetrapropylorthosilicate } & \text { (TPS), } 5 & \text { wt\% }\end{array}$ diphenylmethylsilanol (DPMS) and 15 wt\% of diatomaceous earth. This filler has a large particle size ( $\mu \mathrm{m})$, which thickens the uncured mixture and provides bulk to the cured polymer. The foam is cured by condensation reactions between silanols and silanes groups, with the evolution of hydrogen gas. Condensation reactions between silanols and TPS also contribute to the cross-linking formation with evolution of $n$-propyl alcohol. The condensation reactions are catalyzed by stannous-2-ethylhexanoate (or tin octoate). The cure procedure consisted of mixing 5 wt\% tin octoate into the polysiloxane gumstock, and allowing it to cure at room temperature. The foams were post-cured by heating at $115^{\circ} \mathrm{C}$ for 3.25 hours in air. The average foam density was $0.4 \mathrm{~g} / \mathrm{cm}^{3}$. For the gamma exposure, all samples were placed into sealed aluminum canisters that were evacuated overnight to $10^{-3}$ torr, and backfilled with ultra-high pure nitrogen gas to about 650 torr. Each canister had a volume of approximately $120 \mathrm{~cm}^{3}$ and contained $12 \mathrm{~g}$ of foam. The canisters were exposed to doses of 12.5, 50, and $200 \mathrm{kGy}$ at room temperature and at $1 \mathrm{~Gy} / \mathrm{sec}(10 \mathrm{kGy}$ is equivalent to $1 \mathrm{Mrad})$. Samples were exposed at the gamma irradiation facility (GIF) at Sandia National Laboratories in New Mexico (Co-60 source). In order to detect and quantify the extracted material, samples weighing $100 \mathrm{mg}$ were extracted overnight in $2.5 \mathrm{ml}$ of deuterated chloroform ( $\mathrm{CDCl}_{3} 99.6 \%$ Acros Organics) and analyzed by ${ }^{1} \mathrm{H}$ NMR. In addition to manufacturing SX358 foams, a cross-linked siloxane network was also synthesized by reacting a vinyl-terminated PDMS oligomer with tetrakis(dimethylsilyloxy) silane, $\mathrm{Si}\left(\mathrm{OSiMe}_{2} \mathrm{H}\right)_{4}$ (Gelest products). The molecular weight and molecular weight distribution of the vinyl-terminated PDMS oligomer were determined by GPC, with $M_{n}=5,800$ Daltons and $M_{w}=16,600$ Daltons. A platinum catalyst, Pt (1,3-divinyl-1,1,3,3,tetramethyldisiloxane) as a $0.3 \mathrm{wt} \%$ in xylene, was purchased from Aldrich and used as received (approximately $2 \mathrm{ppm}$ based on amount of vinyl-terminated PDMS) to synthesize the cross-linked network. ${ }^{12}$ The only purpose for synthesizing this material was to better assign the small peaks observed in the solid-state NMR spectrum of irradiated SX358 foams. 
2.2. Gas Analysis. The headspace of each canister containing the irradiated siloxane foams was analyzed using a Finnigan 271 mass spectrometer. Gas samples were collected into small gas bottles from each canister using a small vacuum manifold and injected to the mass spectrometer inlet chamber. The mass spectrometer was calibrated by known mixtures of gases, wherein detector sensitivities to the ions of specific gases are determined. When known mixtures or pure gases were not available for calibration, sensitivity estimates were provided. Initially qualitative spectral scans were performed for each unknown gas sample. Unique peaks were noted for further analysis, and a selection of peaks was scanned quantitatively. The partial pressures of inlet gases that produced the observed ion data were deduced from detector sensitivities. The sum of the molecular gas partial pressures was ratioed to the total measured inlet pressure for each sample, and the results were always within a few percent of $100 \%$. The NIST mass spectral database was consulted for assignment of hydrocarbons in the headspace analyses.

2.3. Chemical Analyses. Solid-state NMR experiments were performed using a Bruker Avance NMR spectrometer operating at $400.13 \mathrm{MHz}$ for ${ }^{1} \mathrm{H}$ using a Bruker (4 mm) Magic Angle Spinning (MAS) probe. The typical $\pi / 2$ pulse width was $3.5 \mu \mathrm{s}$, and about 100 scans were acquired. ${ }^{119} \mathrm{Sn}$ Mössbauer spectroscopy was performed to probe changes in the oxidation state of the tin catalyst. A commercial $\mathrm{Ca}^{119 m} \mathrm{SnO}_{3}$ source provided the $23.87 \mathrm{keV}$ gamma rays. Spectra of the transmission count versus the source absorber Doppler shift rate were fitted using appropriate least square-fitting programs; the statistical errors associated with the fitting procedure are of the order of $\pm 0.02 \mathrm{~mm} / \mathrm{s}$. Continuous wave (CW) EPR spectra were collected to investigate freeradicals using a Bruker model E500 operating in the X-band microwave frequency range equipped with a Bruker SHQE resonator. Spectra were collected at room temperature using a modulation amplitude of $10 \mathrm{G}$, a frequency modulation of $100 \mathrm{kHz}$, an optimum microwave power of $1 \mathrm{~mW}$, a conversion time of $81.92 \mathrm{~ms}$, corresponding time constant of $81.92 \mathrm{~ms}$ to avoid distortion of the signal, and a resolution of 1024 points. Minimal background signals were observed for an empty sample holder. 
2.4. Thermo-analyses. TGA experiments were performed on a Thermal Instrument Q500 model. Samples were heated at $10^{\circ} \mathrm{C} / \mathrm{min}$ under a nitrogen purge. DSC experiments were performed on a TA Instruments Q2000 DSC. The pristine polymer was characterized by a melting temperature at $-48.7^{\circ} \mathrm{C}$ and a glass transition temperature at around $-115^{\circ} \mathrm{C}$.

2.5. Solvent swelling experiments. Average molecular weights between cross-links $\left(\mathrm{M}_{\mathrm{c}}\right)$ were estimated by using a well-known two-step solvent swelling procedure described elsewhere. ${ }^{13}$ Four specimens for each condition were analyzed by this technique. The samples were first swollen in toluene (Fisher Scientific, 99\% purity) and periodically weighed until reaching saturation. After seven days, equilibrium weight was recorded and ammonium hydroxide (Fisher Scientific, $28-30 \mathrm{w} / \mathrm{w} \%$ ) was added to the toluene solution. The samples were then weighed periodically until equilibrium was reached with the toluene/ammonium hydroxide mixture. The additional swelling yield reflects the breaking of the hydrogen-bonding interaction between the surface silanols on the filler and the siloxane backbone of the polymer. The samples were then dried under ambient conditions for seven days and reweighed for the final dry weight. The overall final weight losses from the initial mass were about $3 \%$. The FloryRehner equation was used to estimate the average molecular weight between cross-links for the polymer: ${ }^{14,15}$

$$
M_{c}=\frac{-V_{s} \rho_{p}\left(v_{f}^{1 / 3}-v_{f} / 2\right)}{\ln \left(1-v_{f}\right)+v_{f}+\chi v_{f}^{2}}
$$

Where $V_{s}$ is the molar volume of the solvent $(106.5 \mathrm{~mL} / \mathrm{mol}), \rho_{p}$ is the polymer density, $v_{f}$ is the volume fraction of polymer in the sample at equilibrium swelling, and $\chi=0.465$ is the FloryHuggins polymer-solvent interaction parameter. ${ }^{16}$ The average molecular weight between two neighbors cross-links is inversely proportional to the network cross-link density $\left(v=\rho_{p} / M_{c}\right)$.

2.6. SEM. An FEI Inspect $F$ field emission SEM instrument was used in analyzing the microstructure of the SX358 foams. Surface analysis was carried out by cutting $\approx 1 \mathrm{~cm}^{2}$ samples and placing onto standard mounts with carbon tape facing up. To avoid charging effects, the 
edges of the foam were painted with graphite paint and then sputter coated with $\approx 5 \mathrm{~nm}$ of gold-palladium.

2.7. Mechanical Testing. The samples tested were nominally all the same thickness, density and length, $10.2 \mathrm{~mm}$ in diameter, $0.4 \mathrm{~g} / \mathrm{cm}^{3}$ and $1.045 \mathrm{~mm}$ in length. Quasi-static and low-strainrate compression tests were conducted at strain rates of 0.001 and $0.01 \mathrm{~s}^{-1}$. The low strain rate compression tests were conducted with an MTS model 880 test frame. In addition to the installed load cell and LVDT transducer, additional load measuring devices were used. A $100 \mathrm{lb}$ capacity load cell was used to insure the most accurate measure of the load was made. The control channel of these tests used the extensometer signal. In this case the system has been modified to use a COD (crack opening displacement) calibrated in the "closing" direction to both measure the displacement and as the feedback control channel. This method adds a small additional load due to the spring constant of the device but it can be measured and subtracted from the experimental data if it is a high enough percentage of the overall load signal. Generally the load increase is $\approx 0.75 \mathrm{~N} / 0.5 \mathrm{~mm}$ of displacement, and it is linear over its range of accuracy (0.5" displacement range). This bias load was determined to be $\approx 6.5 \%$ of sample load for the SX358 foam. The analysis of the current results does not subtract away this known bias error since this error is consistent for all tests. The benefit to using this extensometer is that it eliminates system compliance and hysteresis that can be seen in the LDVT signal, thus reducing the uncertainty of the load/displacement for the entire system. The COD device was also used as the control channel because of its accuracy. All tests were conducted with a petroleum jelly lubricant. The experiments were run by placing the samples on optically flat tungsten carbide platens and centered in the loading system. The SX358 material was tested at a strain rate of $0.001 / \mathrm{s}$ to a strain level of $\approx 50 \%$ engineering strain. Three cycles were applied during each test and three samples were tested for repeatability. 


\section{Results and Discussion.}

\subsection{Gas evolution.}

Gases are formed as a result of atom or side-chain abstraction, thus the nature of the gas closely reflects not only the polymer backbone chemical composition, but also the nature of side groups in the repeating unit of the polymer chain. The radiation chemistry of PDMS was first studied by Charlesby who reported that the major volatile products of radiolysis were hydrogen, methane and ethane. ${ }^{1}$ The author proposed cross-link formation to occur due to $\mathrm{C}-\mathrm{H}$ and C-Si bond scission, with no main chain scission. The calculated $G(X)$-value, or the number of cross-links generated per $100 \mathrm{eV}$ of energy absorbed (1/100 eV is equivalent to $1.036 \mathrm{e}-7$ $\mathrm{mol} / \mathrm{J})$, was found to be equal to 3 , and independent of the molecular weight of PDMS. The amount of gas evolved correlated well with the number of cross-links formed at room temperature, which is to be expected since double bond formation cannot occur in silicones, unlike polyethylene. Since then, other studies investigating radiolysis in PDMS at room temperature under inert atmosphere were published in the literature. These show large variations in the yield of the gases products: $\mathrm{G}\left(\mathrm{H}_{2}\right)=0.3$ to $1.25, \mathrm{G}\left(\mathrm{CH}_{4}\right)=1.0$ to $1.3, \mathrm{G}\left(\mathrm{C}_{2} \mathrm{H}_{6}\right)=0.3$ to $0.76 .^{3,17}$ In this work, mass spectroscopy was used to probe the gases formed due to gamma exposure of the RTV foams. Essentially, our results demonstrate that the concentration of volatiles increase linearly with the gamma dose. As expected, the most abundant gases were methane, hydrogen and ethane. Benzene, toluene, propane, butane and cyclic siloxanes such as hexamethylcyclotrisiloxane or D3 $\left((\mathrm{SiO})_{3}\left(\mathrm{CH}_{3}\right)_{6}\right)$ and octamethylcyclotetrasiloxane or D4 $\left(\left((\mathrm{SiO})_{4}\left(\mathrm{CH}_{3}\right)_{8}\right)\right.$ were also found in the headspace, but in much lower concentrations. D3 and D4 were not observed from a control sample in this study, but were observed following extended storage at room (and elevated) temperature without irradiation. In addition, it is worth mentioning that the oxygen concentration in the irradiated samples was of the order of $1 \mathrm{ppm}$, thus ruling out oxidative degradation. Corresponding averaged G-values and the concentrations of the three most abundant gases evolved due to irradiation exposure are listed in Table 1. 
Table 1. Concentrations in ppm of the various gases, benzene, toluene, and siloxane fragments found in the headspace of irradiated and control SX358 foams. Siloxane fragments are likely due to outgassing. (G-values are given in $10^{-7} \mathrm{~mol} / \mathrm{J}$ ).

\begin{tabular}{lcccccc}
\hline Dose (kGy) & $\mathbf{H}_{\mathbf{2}}$ & $\mathbf{C H}_{\mathbf{4}}$ & $\mathbf{C}_{\mathbf{2}} \mathbf{H}_{\mathbf{6}}$ & Si radicals & Benzene & Toluene \\
\hline control & 19 & 0.7 & $<0.7$ & $<0.2$ & 0.1 & $<0.05$ \\
12.5 & 891 & 1500 & 347 & 0.3 & 10.2 & 0.3 \\
50 & 4300 & 9300 & 1500 & 1.6 & 40.5 & 2.0 \\
200 & 18300 & 41000 & 5800 & 6.7 & 119.7 & 9.1 \\
\hline G-value & $\mathbf{0 . 3 9}$ & $\mathbf{0 . 8 3}$ & $\mathbf{0 . 1 3}$ & & & \\
\hline
\end{tabular}

The total $G(X)$-value for the RTV foam is estimated to be of the order of 1.3. It is interesting to compare this result with others published in the literature for PDMS-based materials with varying amounts of phenyl groups. Our RTV foam contains about $5 \mathrm{wt} \%$ of DPMS, but these phenyl groups are not part of the polysiloxane chain backbone. Delides investigated the extent of protection afforded by the incorporation of phenyl groups through molecular weights and solubility studies. ${ }^{18}$ The author found that aromatic substitution in the polymer backbone remarkably reduced the cross-linking efficiency, and provided protection against radiolysis that increased almost exponentially with increasing phenyl concentration. Depending on the phenyl concentration, $G(X)$ values were found to vary from 2.3 for PDMS containing no phenyl groups to about 1.09 for polymers containing about $21 \%$ phenyl groups. In addition, radiation stability depended not only on the total aromatic content, but also on the type and position of the aromatic group. Thus, co-located aromatic substituents, such as diphenyl are expected to confer greater stability than a single phenyl group.

\subsection{Changes in Chemistry.}

Infrared studies performed on irradiated PDMS by Miller and Bueche reported the formation of $\mathrm{Si}-\mathrm{H}$ structures, whereas Belova proposed the formation of $\mathrm{Si}-\mathrm{O}$ bonds as 
well. $^{3,17,19}$ Miller estimated the yields for the silylmethylene cross-links ( $\equiv \mathrm{SiCH}_{2} \mathrm{Si} \equiv$ ) to be 1.8 , silylethylene cross-links ( $\equiv \mathrm{SiCH}_{2} \mathrm{CH}_{2} \mathrm{Si} \equiv$ ) to be 0.5 , and by a bromination method, Si-Si H-type cross-links were also observed with a yield value of $1.1 .^{3}$ More recently, Hill and co-workers identified new structural units consistent with main chain scission and cross-linking through both $\mathrm{H}$-linking and $\mathrm{Y}$-linking reactions as shown in Figure $1 .{ }^{20-22}$ The authors used ${ }^{29} \mathrm{Si}$ and ${ }^{13} \mathrm{C}$ NMR to study the structural changes in gamma exposed PDMS under vacuum at $303 \mathrm{~K}$. The results for $G$-values at various absorbed doses indicated $G(S)=1.3 \pm 0.2$ for chain scission, $G(H)$ $=0.34 \pm 0.02$ for $\mathrm{H}$-linking, and $G(Y)=1.70 \pm 0.09$ for $Y$-linking. Thus cross-linking predominates over scission for radiolysis of PDMS under these conditions and by contrast with previous studies, Y-links were shown to be the most important form of cross-links.

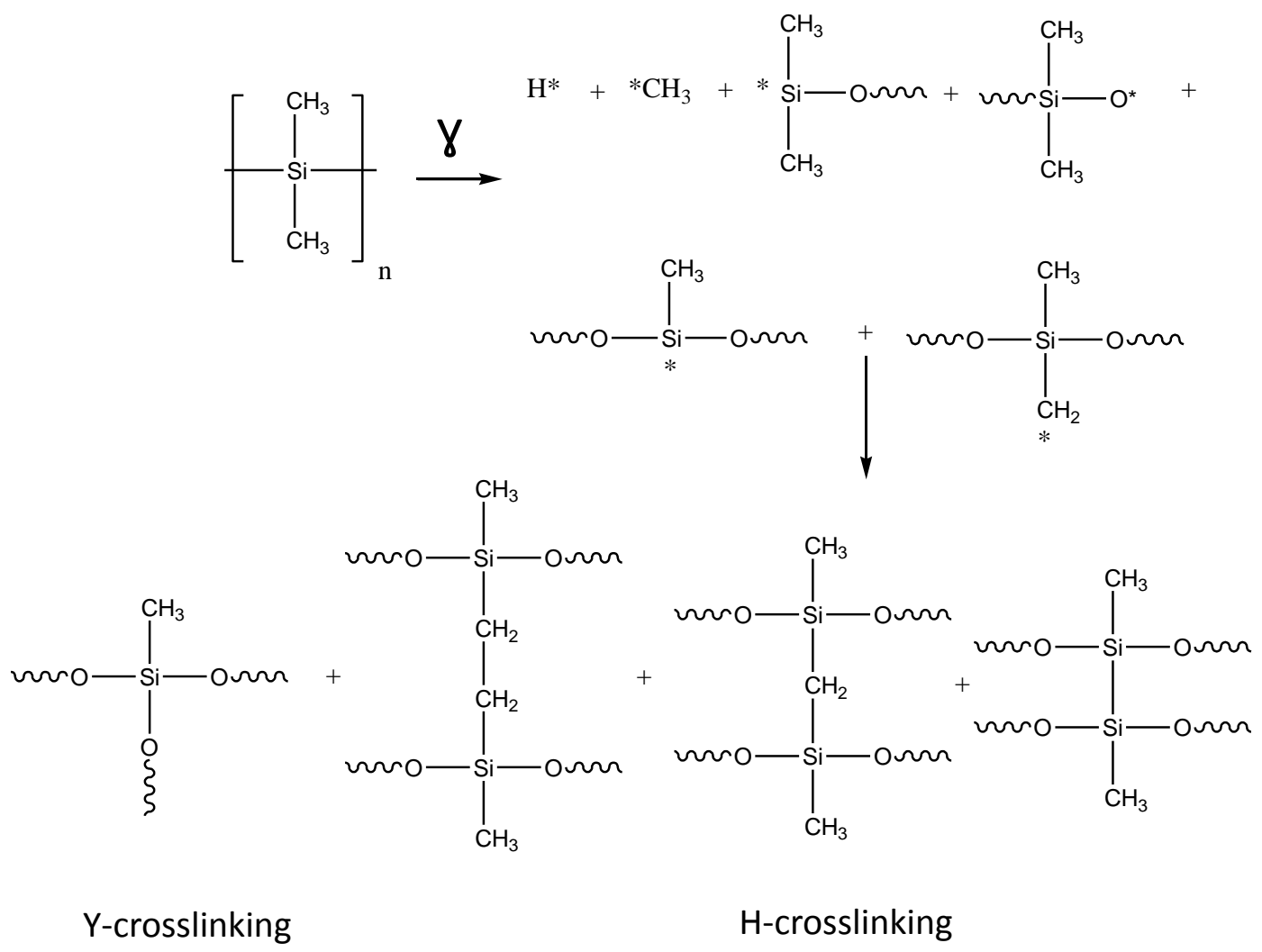

Figure 1. Scheme showing radiation induced radicals due to chain scission; radiation induced macroradicals due to methyl or hydrogen abstraction; and irradiation induced $\mathrm{Y}$ and $\mathrm{H}$ crosslinks produced when PDMS is exposed to gamma rays under vacuum. ${ }^{10}$ 
In this work, changes in the chemistry of irradiated RTV foams were probed by FT-IR, NMR, and Mössbauer spectroscopy. In general, the detection of radiochemical damage in PDMS-based materials is possible only when large gamma doses are employed. The low sensitivity of these spectroscopic techniques coupled together with the low gamma doses employed here, renders the chemical characterization of irradiated polymers difficult to carry out since very few chemical changes are expected to occur in the material. Thus, it is not surprising that no chemical changes were observed by FT-IR and very subtle ones were detected by solid-state NMR. In the studies performed by Hill and co-workers, where the authors used solid-state ${ }^{13} \mathrm{C}$ and ${ }^{29} \mathrm{Si}$ NMR to probe irradiation damage in PDMS, the doses were above 1.1 MGy, nearly five times higher than the highest dose used in the present study. To try to overcome this shortcoming, we applied solid-state ${ }^{1} \mathrm{H}$ NMR to characterize radiolysis in RTV foams. ${ }^{1} \mathrm{H}$ is the most NMR sensitive nucleus, but it has a narrow chemical shift range and usually yields broad signals in the solid-state. In addition, solid-state ${ }^{1} \mathrm{H}$ NMR does not afford the identification of different chemical species as well as ${ }^{29} \mathrm{Si}$ and ${ }^{13} \mathrm{C} \mathrm{NMR}$. Nonetheless, polymer chains in PDMS have high chain mobility and therefore, the ${ }^{1} \mathrm{H} N M R$ signals are relatively narrow and easy to observe. Figure 2 reveals the ${ }^{1} \mathrm{H}$ MAS NMR spectra obtained for irradiated SX358 foams. An additional sample was exposed to $300 \mathrm{kGy}$ so changes in the spectrum with dose could become more apparent. A set of large peaks are easily detected, one close to $0 \mathrm{ppm}$ which is associated with methyl groups from PDMS, and a double peak centered at $7.3 \mathrm{ppm}$ which is associated with diphenyl groups. Furthermore, a set of small peaks in the baseline are associated with protons from the residual catalyst, unreacted silane groups (4.7 ppm), and silanol groups on the surface of the filler and/or physi-absorbed water (3.7 ppm). Interestingly, a couple of very small peaks whose intensity increases with the gamma dose are also observed. These are detected at 1.1 and 0.90 ppm in the irradiated samples, but not in the control sample. As for comparison, the ${ }^{1} \mathrm{H}$ MAS NMR spectrum of a sample formed by hydrosilylation of vinyl-terminated PDMS is also shown. The spectrum is dominated by the large peak at about $0.05 \mathrm{ppm}$, which is associated with protons from methyl groups in the PDMS chain. Methyl and methylene protons that are next near-neighbors to cross-links are also observed in the spectrum at 0.49 and $1.1 \mathrm{ppm}$; respectively. Thus, the peak at about $1.1 \mathrm{ppm}$ 
observed in the spectrum of the irradiated foams is likely associated with methylene protons resulting from cross-link formation.

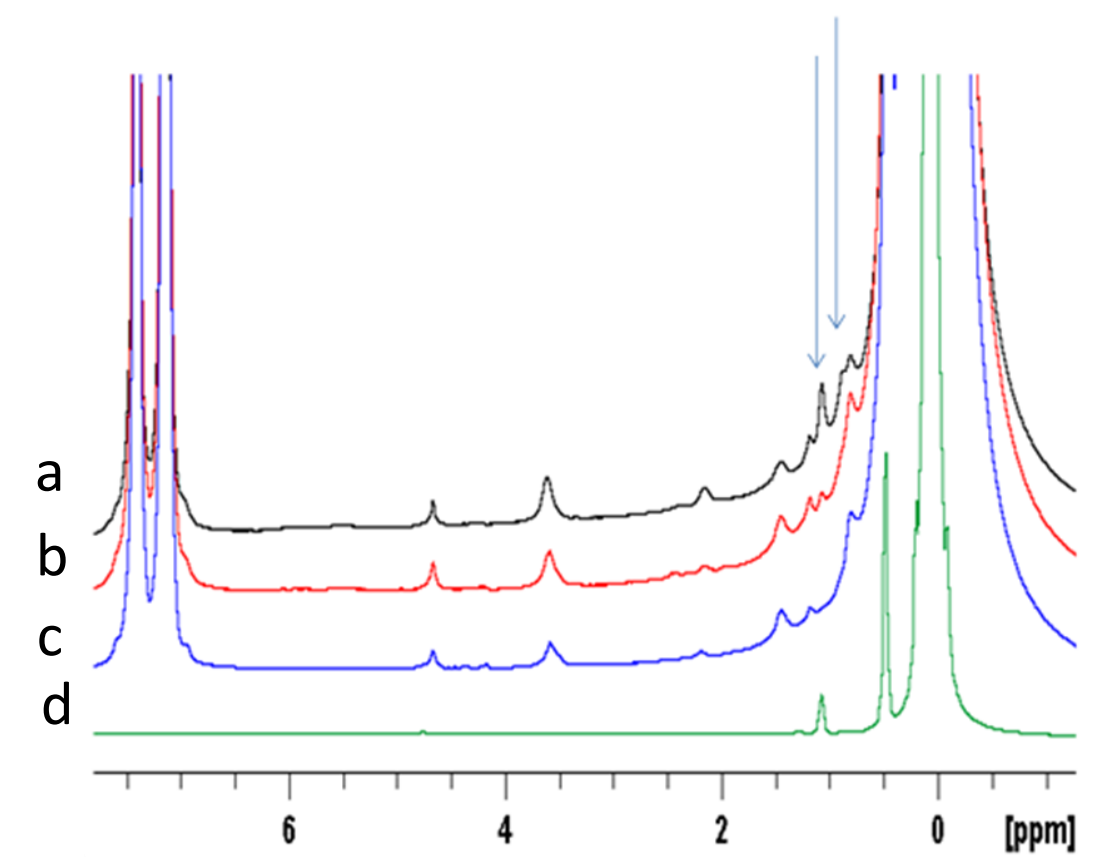

Figure 2. ${ }^{1} \mathrm{H}$ MAS NMR spectra of SX358 foams irradiated at (a)300 kGy, (b) $200 \mathrm{kGy}$, and (c) control. The arrows indicate small new peaks at 1.1 and $0.90 \mathrm{ppm}$ that increase in intensity with gamma exposure. The NMR spectrum of the sample formed by hydrosilylation of vinylterminated PDMS is also shown here (d).

Extracted material from the RTV foam was analyzed by liquid-state ${ }^{1} H$ NMR spectroscopy with the aim of detecting chemical signatures associated with radiolysis. Control and irradiated SX358 foams were extracted using deuterated chloroform to isolate soluble compounds from the polymer network such as unreacted starting materials, fragments due to chain scission, and other impurities. Extracted material was found to be chemically similar from sample to sample and quantitatively comparable, suggesting that similar amounts of chemical species were extracted from control and irradiated foams (Figure 1 in Supporting Information). 
The polysiloxane foam investigated in this study was cured by stannous octoate, an organotin catalyst generally used to promote the condensation chemistry of dihydroxy terminated polydimethylsiloxane with alkoxysilanes. Residues of the tin(II) catalyst are associated with promoting aging of polysiloxane foams as evidenced by changes in the mechanical response of the material like load retention properties. ${ }^{23,24}$ Earlier data reported in the literature demonstrate that ${ }^{119} \mathrm{Sn}$ Mössbauer spectroscopy is a useful technique to probe the nature of the tin residues that remain in the RTV foam. ${ }^{25,26}$ This method detects differences between the chemical environments of a gamma emitter and an absorber, where a shift of the spectral line occurs due to the different electronic environments interacting with each atom's nuclear energy levels. In addition, the interaction between the nuclear quadrupole moment of ${ }^{119} \mathrm{Sn}$ and an electric field gradient at the nucleus produces a doublet splitting of the resonance line or quadrupole splitting. ${ }^{119} \mathrm{Sn}$ Mössbauer spectroscopy shows that the tin catalyst slowly oxidizes to form tin(IV) residues, which are thought to be $\mathrm{SnO}_{2}$. The oxidation process takes place in the neat tin catalyst as well as for tin residues that remain in the RTV foam. ${ }^{9}$ In the present work, this technique was applied to investigate possible chemical changes in the oxidation state of tin residues due to gamma exposure in a nitrogen atmosphere as well as in air. Figure 3 shows ${ }^{119}$ Sn Mössbauer data obtained for the pristine tin(II) catalyst, pristine RTV foam and RTV foam after being exposed to gamma irradiation in air and under nitrogen. The Mössbauer spectrum of the neat catalyst shows mostly tin (II) species, with small amounts of tin (IV) species. Typical relative tin (IV) amounts in pristine stannous octoate vary between 5 to $10 \%$. The spectrum of the pristine foam shows the presence of tin(II) species centered at 3.7 $\mathrm{mm} / \mathrm{s}$ and tin(IV) species close to $0 \mathrm{~mm} / \mathrm{s}$; the same result is obtained when the foam is irradiated in a nitrogen environment. The relative amounts of tin(II) species remained unchanged after gamma exposure at about 32\% ( $\pm 1 \%)$. However, when the foam is exposed to gamma irradiation in air, only the tin(IV) species are detected. Thus, there is complete oxidation of $\operatorname{tin}(\mathrm{II})$ residues induced by the ionizing irradiation. The same effect was observed for the neat catalyst. Under a nitrogen atmosphere, no oxidation occurs due to the ionizing exposure. We also note that the chemical shift and electric field gradients observed for the tin(IV) and for the 
tin(II) sites in all samples studied show that the local environment at each of the two types of sites change very little with various treatments of the prepared foams.
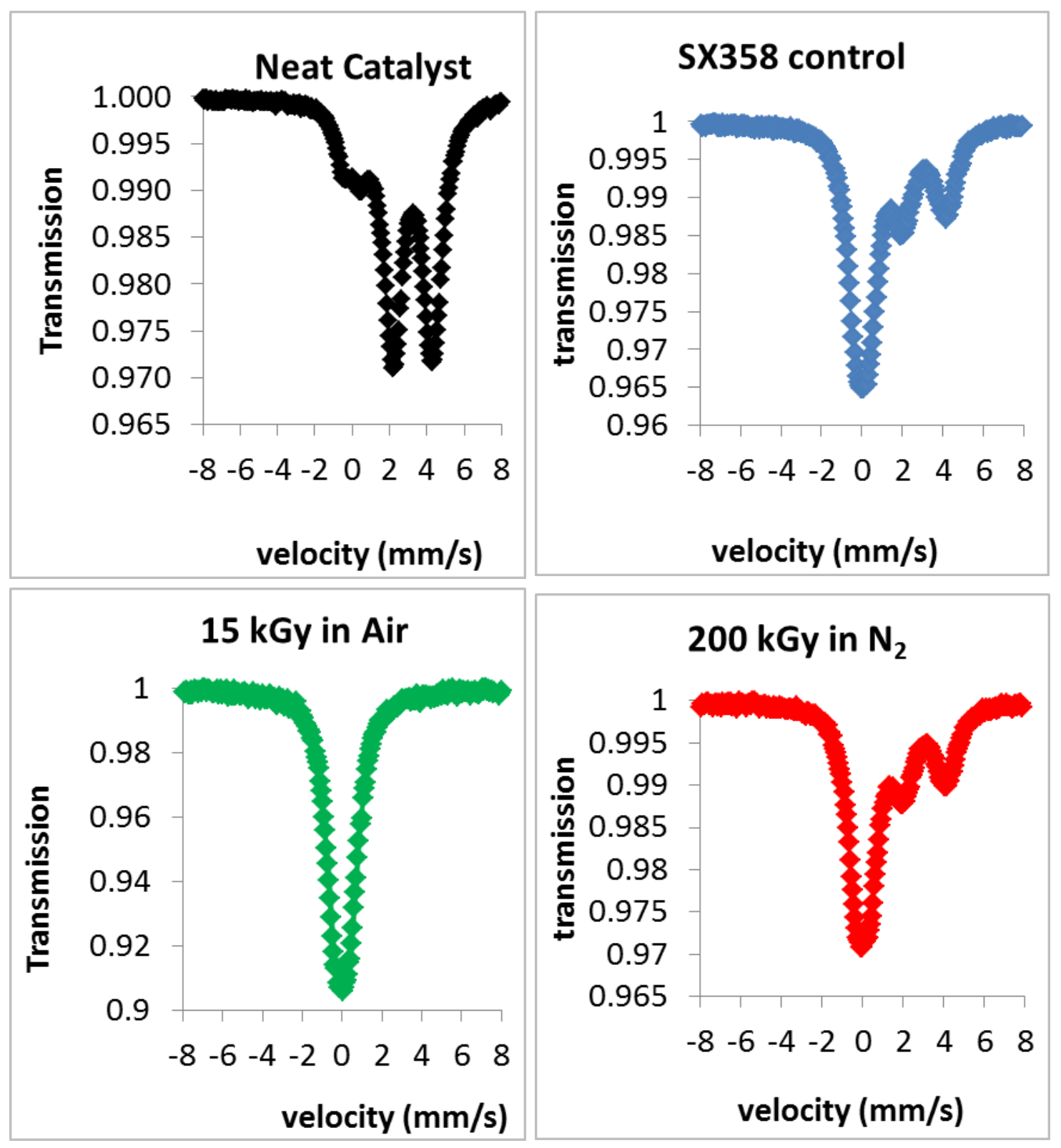

Figure $3 .{ }^{119}$ Sn Mössbauer spectra obtained for the pristine tin octoate catalyst (black trace), residual tin in the pristine polysiloxane foam (blue trace), then after the RTV foam was exposed to $15 \mathrm{kGy}$ in air (green trace), or after the foam was exposed to $200 \mathrm{kGy}$ under nitrogen atmosphere (red trace). 
EPR spectroscopy was performed to monitor the formation of any stable paramagnetic species that were formed during the irradiation of the SX358 foam. CW EPR spectra collected by Patel et al. on similar RTV foam (RTV-5370) revealed the formation of an irradiation induced paramagnetic species. ${ }^{7}$ The EPR signal intensity for RTV-5370 showed a dependence on the radiation dose, increasing dramatically until a dose of $10 \mathrm{kGy}$, where it began to level off. The origin of the narrow EPR spectrum was assigned to a species that formed in the silica filler due to trapped paramagnetic species in the silica matrix. In comparison, the spectra of the SX358 foam revealed a much broader signal $(\approx 3000 \mathrm{G})$ due to a paramagnetic species that did not have any dependence on the irradiation dose (Figure 2 in Supporting Information). Due to the very broad and intense feature centered at $g=2.011$, it is difficult to resolve any narrow signals, which would suggest the presence of a radical species. Nonetheless, we cannot rule out the presence of an irradiation-induced paramagnetic species that maybe underlying the broad EPR spectrum.

\subsection{Changes in Thermal Properties.}

The thermal stability of the irradiated RTV foams was probed by TGA experiments. Table 2 lists the temperatures in which there is a 5\% decrease of the initial mass. The control sample lost $5 \%$ of its initial mass when the temperature reached $344{ }^{\circ} \mathrm{C}$, whereas the gamma exposed samples lost the same amount of mass at higher temperatures. The onset of thermal decomposition increased with the exposure dose, indicating that the polymer became more thermally stable after irradiation.

DSC was used to examine changes induced in crystallization phenomena by exposure to gamma irradiation. Crystallinity may influence many mechanical properties such as modulus, yield stress, and creep, making it an important feature to probe. DSC thermograms of irradiated and control samples are shown in Figure 4. The glass transition temperature or $T_{\mathrm{g}}$ is seen at about $-115^{\circ} \mathrm{C}$, and it shows little dependence on the gamma dose. An exothermic peak in the cooling cycle and an endotherm peak in the heating cycle are seen in the thermograms as well. 
There is a shift towards lower crystallization and melt temperatures with increasing radiation dose. The melt temperature decreases from $-48.7^{\circ} \mathrm{C}$ for the control sample to $-54{ }^{\circ} \mathrm{C}$ for the foam exposed to $200 \mathrm{kGy}$ (see Table 2). In addition, the $200 \mathrm{kGy}$ sample seems to reveal a second broad peak at lower temperatures (close to $T_{\mathrm{g}}$ ) for both crystallization and melt events. This behavior could be due to the presence of two different crystalline domains. The DSC results are consistent with cross-linking formation due to radiolysis. In other words, cross-link formation may decrease the level of crystallinity and change the nature of the crystallites by topologically hindering the chains to readily pack to form ordered structures. Roland and Aronso estimated that each cross-link may prevent about 8 adjoining chain units from joining into the crystal phase. ${ }^{27}$ Thus, cross-linking decreases the degree of crystallinity as well as the stability of the crystal phase.
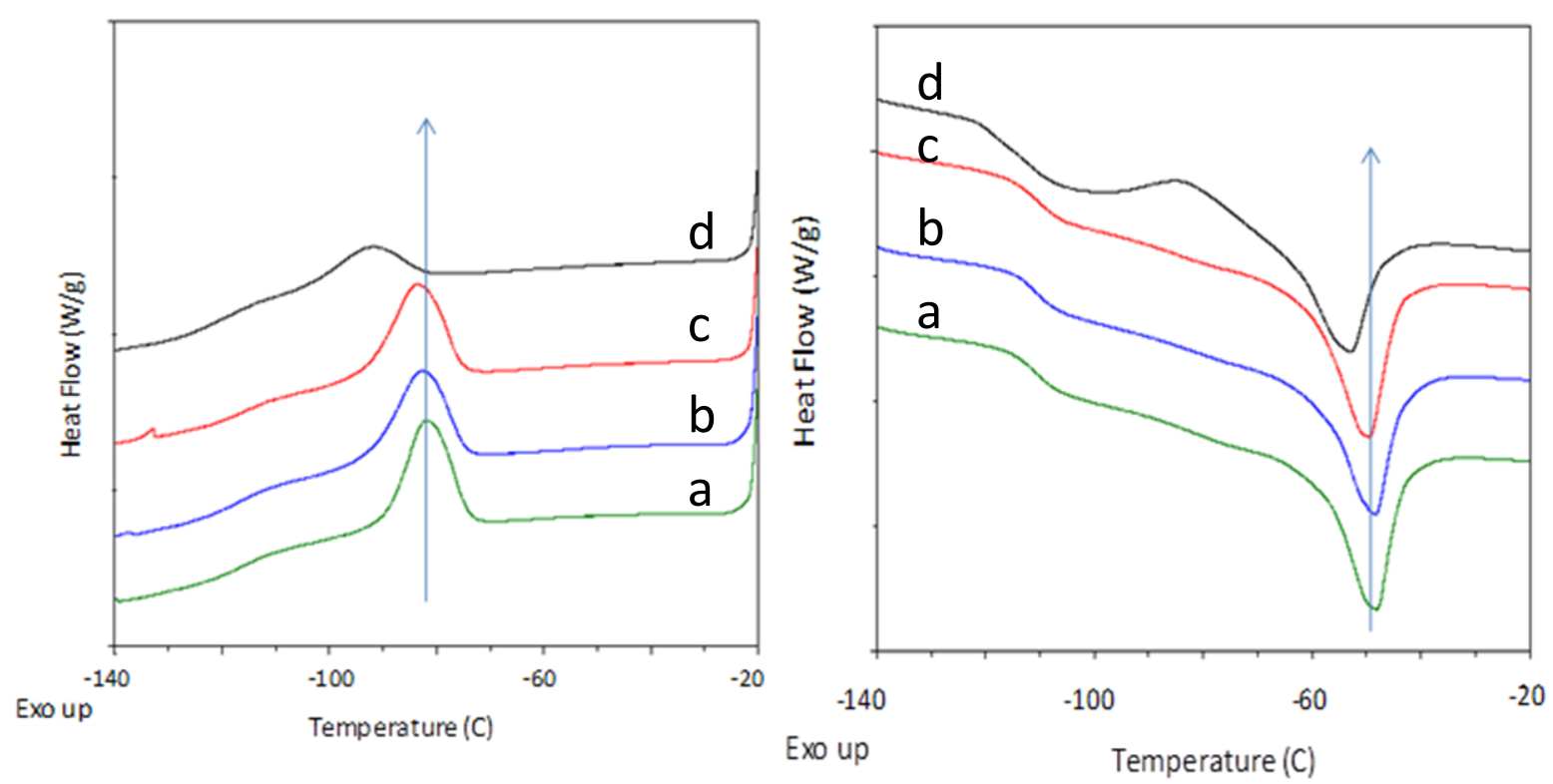

Figure 4. DSC cooling (left) and heating (right) thermograms for (a) control and irradiated SX358 foams, (b)12.5 kGy, (c) $50 \mathrm{kGy}$, and (d) $200 \mathrm{kGy}$. (Arrows are just to illustrate the shifting of crystallization and melting events with gamma exposure). 
Table 2. Onset temperatures for which the foams lose $5 \%$ of their initial mass as measured by TGA, and melting temperatures observed by DSC.

\begin{tabular}{ccc}
\hline Dose (kGy) & Onset & Melting Temperature \\
& Temperature $\left({ }^{\circ} \mathrm{C}\right)$ & $\left({ }^{\circ} \mathrm{C}\right)$ \\
\hline control & 344.2 & -48.7 \\
\hline 12.5 & 347.2 & -48.7 \\
\hline 50 & 348.7 & -50.2 \\
\hline 200 & 351.0 & -54.0 \\
\hline
\end{tabular}

\subsection{Changes in Microstructure.}

Wei and co-workers used SEM techniques to investigate the effects of gamma irradiation on the microstructure of siloxane foam containing phenyl groups. ${ }^{8}$ The authors found that the open and spherical microstructure collapsed after being exposed to 1 MGy dose. In the present work, the microstructure of RTV foams was investigated by AFM and SEM techniques. AFM images of irradiated and control foams show no significant changes in morphology and roughness due to gamma exposure (see Figure 3 in the Supporting Information). Similar results were observed by SEM and are illustrated in Figure 5. Analysis of the top surface of the RTV foam reveals a rough, bubble-like skin layer and a smooth uniform skin layer on the bottom side. The changes observed in the images are attributed to the nonhomogeneity of the foam. There were no systematic changes that could unambiguously be attributed to radiation exposure. 


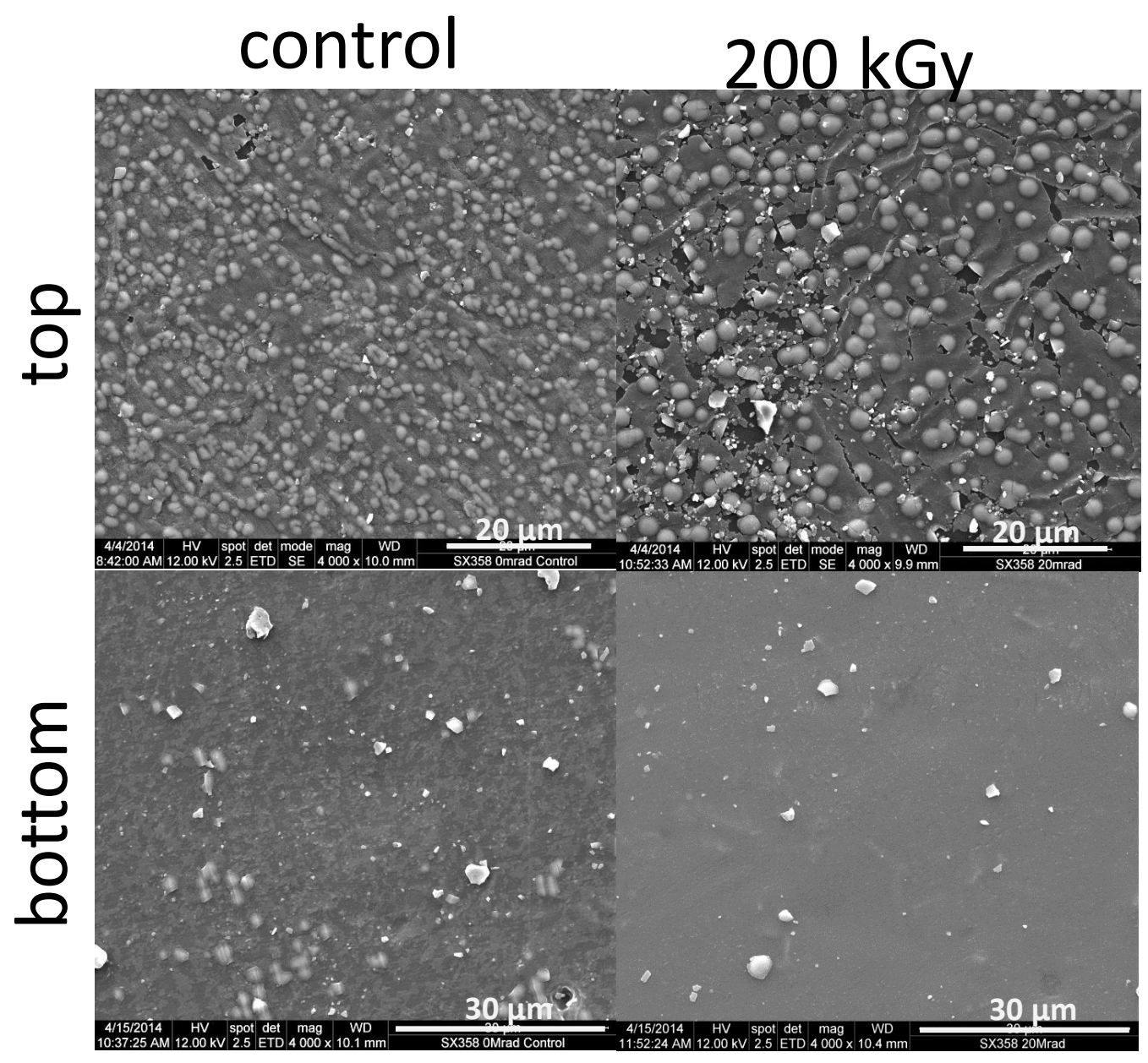

Figure 5. SEM analysis of control and $200 \mathrm{kGy}$ irradiate foam on the top and bottom surfaces.

Intriguingly, tin formations on the inside surfaces of the voids were observed in the cross sections of the RTV foams as showed in Figure 6. It is interesting to compare our results with those obtained by Patel and co-workers on RTV-5370 foam. ${ }^{28}$ The authors assessed the distribution of tin particles throughout the foam using X-ray fluorescence (XRF). They found that the tin distribution is non-uniform with higher concentrations associated with the skin layer, as well as small amounts of tin particles also being present in cell walls or struts. Tin formations are thought to be related to $\mathrm{SnO}_{2}$ particles, which resulted from the tin catalyst oxidation. 

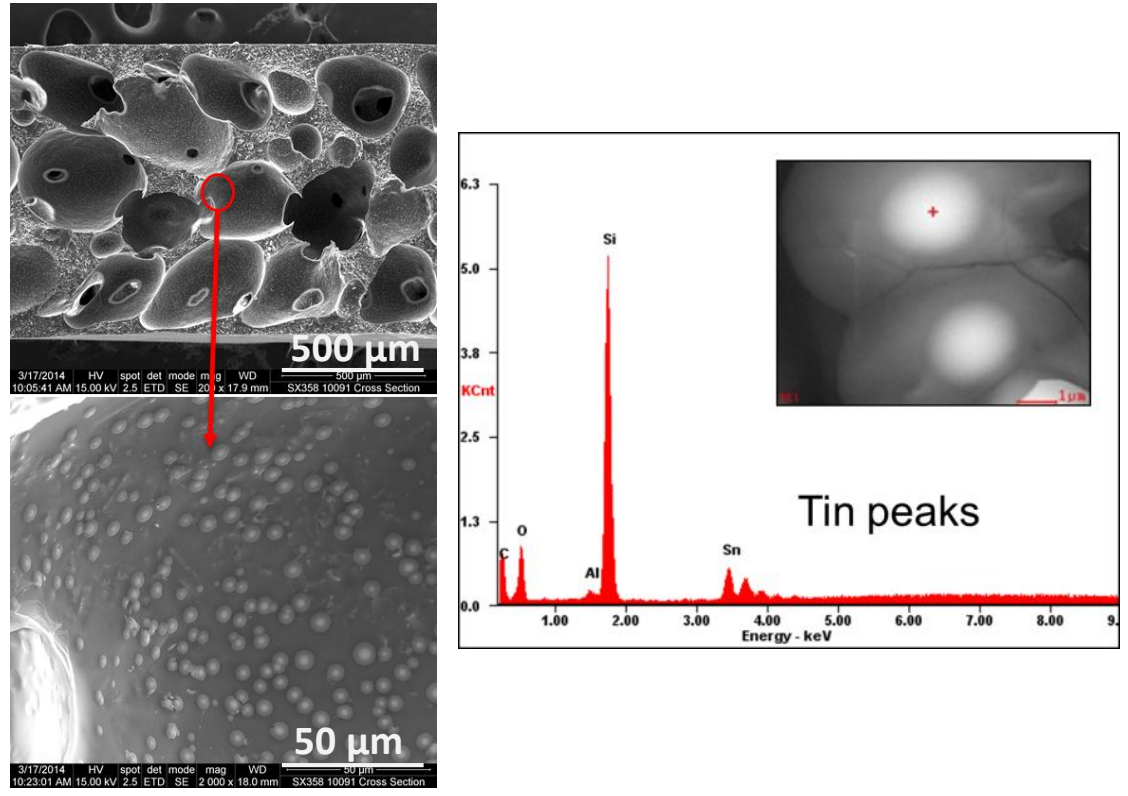

Figure 6. Tin formations as seen by SEM and EDS in the control foam. The area probed by EDS is the inside surface of the voids.

\subsection{Changes in Solvent Uptake.}

The cross-link density is an important property that influences key characteristics of cured rubber. For instance, increasing in cross-link density leads to considerable increase in elastic modulus and hardness, accompanied by a reduction in elongation and permanent set. ${ }^{29}$ Cross-link density can be estimated from different methods with solvent swelling being one of the most common ones. The well-known Flory-Rehner theory relates solvent swelling values to cross-link density through the polymer-solvent interaction parameter and it has been largely used in the rubber industry. However, equilibrium swelling of RTV foams involves not only solvent uptake by the polymer network, but also by the microstructure. In the present work, no corrections were made for the solvent uptake by the porous structure. It was assumed that, since the density of the irradiated foams did not change with gamma exposure, then solvent uptake due to the porous nature of the material is the same for all samples. Figure 7 shows swelling results obtained for irradiated and control SX358 samples. We estimated the molecular 
weight between cross-links, $M_{c}$, using solvent uptake in the toluene/ammonium hydroxide swelling region. This swelling region probes chain entanglements and covalent cross-links, assuming that the ammonium hydroxide broke the hydrogen-bonding interaction between polymer chains and filler particles. Our data indicate a linear decrease in $M_{\mathrm{c}}$ with increasing irradiation dose, suggesting that extra cross-links were formed due to gamma exposure. There is a $17 \%$ decrease in the average $M_{c}$ for the sample exposed to $200 \mathrm{kGy}$ when compared to the control sample.

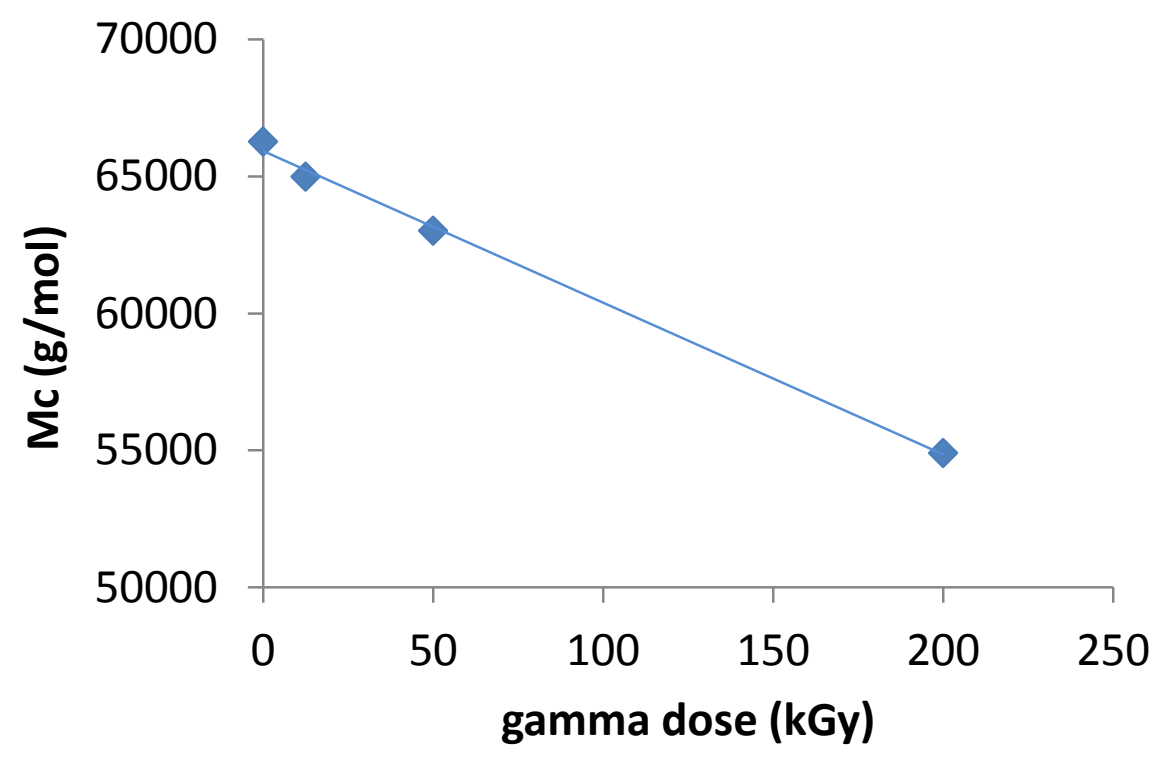

Figure 7. Results of solvent swelling experiments on irradiated SX358 foams. There is a decrease in the molecular weight between covalent cross-links with increasing radiation dose.

\subsection{Changes in Mechanical Properties.}

Three tests were run on each of the four RTV materials. The data were analyzed and one curve for each dose was selected to represent the "typical" behavior for each lot of material. Figure 8 shows a gradual stiffening of the polymer as exposure to gamma dose increases. This is likely an indication that the polymer is stiffening due to increasing in cross link density, although increasing in polymer-filler interactions cannot be ruled out. The general behavior for all lots of material shows a Mullins effect where a decrease in strength is observed after the first cycle of 
loading. ${ }^{30}$ Subsequent loading cycles are nearly identical to each other indicating that polymeric structure has reoriented or "damaged" itself during the first cycle and reached a steady state.

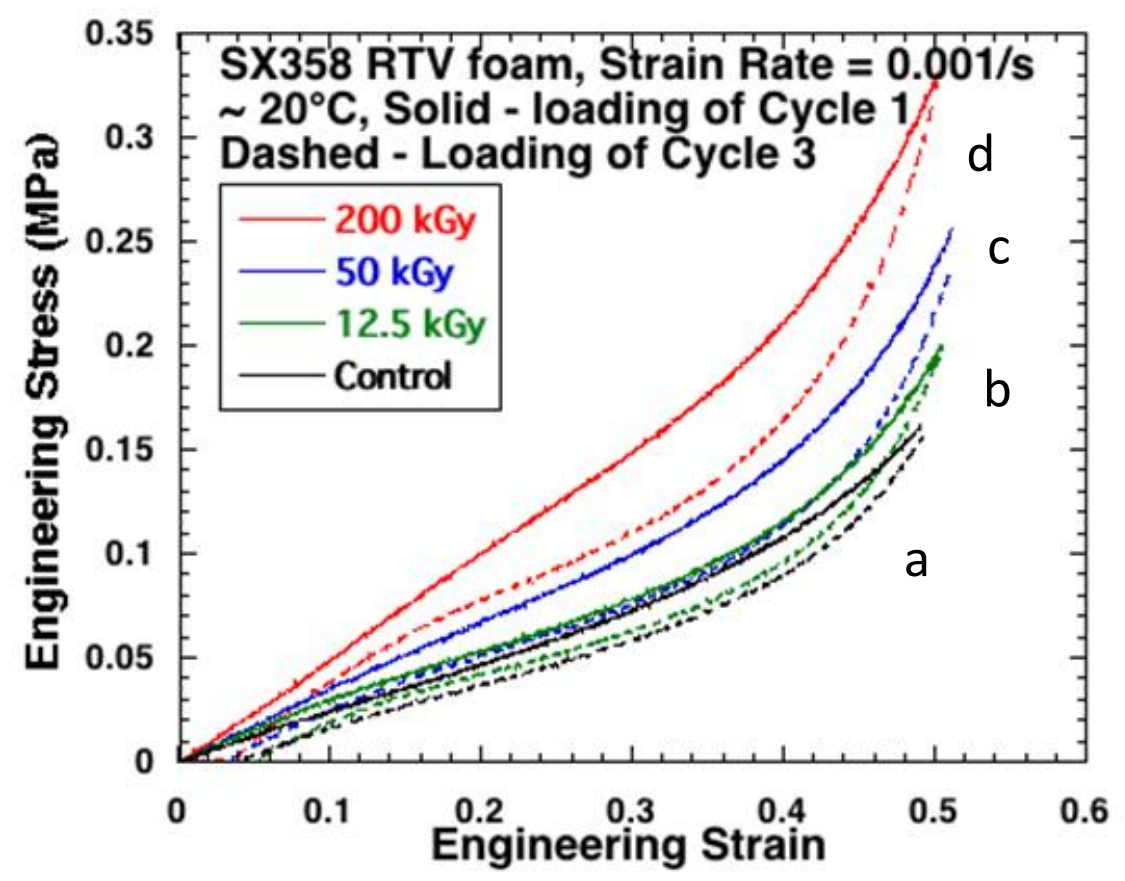

Figure 8. Engineering stress-engineering strain response showing the increasing strength with increased exposure of control and irradiated SX358 foams, (a) control, (b) 12.5 kGy, (c) 50 kGy , and (d) $200 \mathrm{kGy}$.

An interesting observation about the material stiffness is that the first loading seems to have less variation in stiffness with strain. The stiffness was measured by finding the slope of the curve from 0 strain to the value reported (i.e.10\%, 20\%, 30\% and $40 \%$ ). This behavior is similar to the response of a visco-elastic material. During the first cycle there is some damage introduced into the polymer. This is evidenced by the unrecovered strain seen in the material after unloading. Reloading of the sample occurs at the point when the unrecovered strain is exceeded. Due to the damage in the material, it is possible to have an increase in stiffness without exceeding the initial load. This behavior can also be seen when comparing the stiffness plots for cycle 1 and cycle 3 . An observation that can be made about the material stiffness 
between the first and subsequent cycles is that there seems to be a larger drop in stiffness for the $200 \mathrm{kGy}$ and $50 \mathrm{kGy}$ dose samples as compared with the materials exposed to lower dose (Figure 9). This behavior is subtle for the low dose sample as compared to the control.

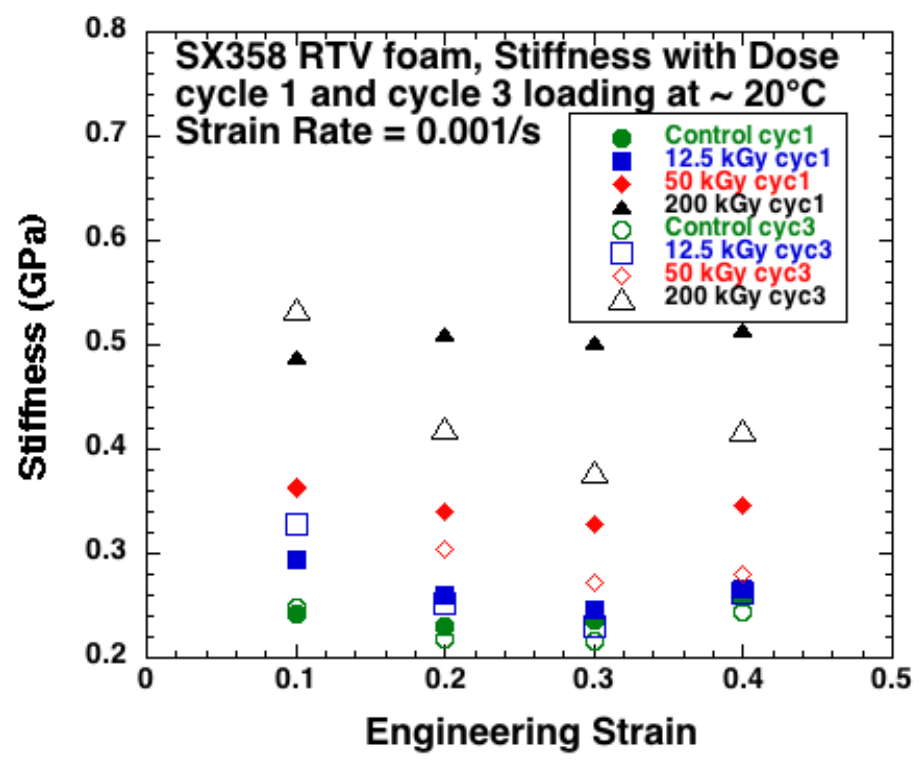

Figure 9. Combined stiffness plot obtained for cycle 1 and cycle 3 showing the change in stiffness after the first cycle.

\section{Conclusions.}

Our data have demonstrated that an RTV polysiloxane foam known as SX358 is susceptible to radiation damage when exposed to moderate gamma doses. Increasing exposure to gamma irradiation under a nitrogen atmosphere caused an increase in the evolution of methane, hydrogen, and ethane as evidenced by mass spectroscopy analysis of the headspace. Small concentrations of D3 and D4 cyclic oligomers were detected as well, but were attributed to outgassing of the sample. Thermal properties of the irradiated foams changed when compared with the control. TGA experiments showed that the onset of the thermal decomposition occurred at higher temperatures, whereas less crystallization and lower melting temperature were evidenced by DSC results. Routine characterization methods such as FT-IR exhibited very low sensitivity in order to detect radiation-induced damage. On the other hand, 
solid-state NMR experiments provided some insights on the chemical degradation of irradiated foams. NMR data indicated that irradiated foams displayed slight changes in chemistry, which may be associated with the presence of silylmethylene and silylethylene groups. Mössbauer spectroscopy showed no changes in the oxidation state of tin catalyst residues when the RTV foam is exposed to gamma irradiation under a nitrogen atmosphere, and complete oxidation when the foam is irradiated in air. The same result was observed for the neat tin catalyst. Solvent swelling experiments revealed a decrease in solvent uptake which was explained by a slight increase in the cross-link density. Microscopy imaging techniques detected no changes to the porous microstructure. EPR spectroscopy observed a broad paramagnetic signal that was attributed to iron species present in the filler. No irradiation induced long-lived paramagnetic species were observed. Mechanical compression testing revealed that the irradiated foams were stiffener than the control ones. All these results taken together coherently indicate that cross-linking is the dominant radiolysis mechanism induced in RTV foams when exposed to moderate radiation doses in inert atmospheres.

Acknowledgments: We thank Don Hanson and Maryla Wasiolek from Sandia National Laboratories for their help with experiments performed at the GIF. This work was funded by the Enhanced Surveillance Campaign, and the US Department of Energy's National Nuclear Security Administration under contract DE-AC52-06NA25396. 


\section{References.}

(1) Charlesby, A. Proc $R$ Soc L Ser-A 1955, 230, 120.

(2) Stpierre, L. E.; Dewhurst, H. A.; Bueche, A. M. J Polym Sci 1959, 36, 105.

(3) Miller, A. A. J Am Chem Soc 1960, 82, 3519.

(4) Warrick, E. L. Ind Eng Chem 1955, 47, 2388.

(5) Basfar, A. A. Rad Phys Chem 1997, 50, 607.

(6) Maxwell, R. S.; Cohenour, R.; Sung, W.; Solyom, D.; Patel, M. Polym Degrad Stab 2003, $80,443$.

(7) Patel, M.; Morrell, P. R.; Murphy, J. J.; Skinner, A.; Maxwell, R. S. Polym Degrad Stab 2006, 91, 406.

(8) Wei, H.; Fu, Y. B.; Wang, C. Y.; Xu, Y. S.; Bian, Z. S. Rad Phys Chem 2002, 64, 229.

(9) Labouriau, A.; Cox, J. D.; Schoonover, J. R.; Patterson, B. M.; Havrilla, G. J.; Stephens, T.; Taylor, D. Polym Degrad Stab 2007, 92, 414.

(10) Satti, A. J.; Andreucetti, N. A.; Ressia, J. A.; Vallat, M. F.; Sarmoria, C.; Valles, E. M. Eur Polym J 2008, 44, 1548.

(11) Menhofer, H.; Zluticky, J.; Heusinger, H. Rad Phys Chem 1989, 33, 561.

(12) Urayama, K.; Kawamura, T.; Kohjiya, S. Macromolecules 2001, 34, 8261.

(13) Vondracek, P.; Schatz, M. J Appl Polym Sci 1979, 23, 2681.

(14) Flory, P. J.; Rehner, J. J Chem Phys 1943, 11, 512.

(15) Flory, P. J.; Rehner, J. J Chem Phys 1943, 11, 521.

(16) Bueche, A. M. J Polym Sci 1955, 15, 97.

(17) Belova, V. V.; Pridachina, N. N.; Popova, A. I.; Serenkov, V. I. Radiolysis of Polysiloxane Resin, 1971.

(18) Delides, C. G. Rad Phys Chem 1980, 16, 345.

(19) Bueche, A. M. J Polym Sci 1956, 19, 297.

(20) Hill, D. J. T.; Preston, C. M. L.; Whittaker, A. K.; Hunt, S. M. Macromol Symp 2000, 156, 95.

(21) Hill, D. J. T.; Preston, C. M. L.; Salisbury, D. J.; Whittaker, A. K. Rad Phys Chem 2001, 62, 11.

(22) Hill, D. J. T.; Preston, C. M. L.; Whittaker, A. K. Polymer 2002, 43, 1051.

(23) Patel, M.; Morrell, P. R.; Skinner, A. R. Macromol Symp 2002, 180, 109.

(24) Patel, M.; Chinn, S.; Maxwell, R. S.; Wilson, T. S.; Birdsell, S. A. Polym Degrad Stab 2010, 95, 2499.

(25) Labouriau, A.; Taylor, D.; Stephens, T. S.; Pasternak, M. Polym Degrad Stab 2006, 91, 1896.

(26) Patel, M.; Skinner, A. R.; Chaudhry, A.; Billingham, N. C.; Mahieu, B. Polym Degrad Stab 2004, 83, 157.

(27) Roland, C. M.; Aronson, C. A. Polym Bull 2000, 45, 439.

(28) Patel, M.; Boxwell, J. L.; Black, S.; Mills, D.; Pitts, S. Polym Test 2014, $39,86$.

(29) Lee, S.; Pawlowski, H.; Coran, A. Y. Rubber Chem Technol 1994, 67, 854.

(30) Mullins, L. Rubber Chem Technol 1948, 21, 281. 